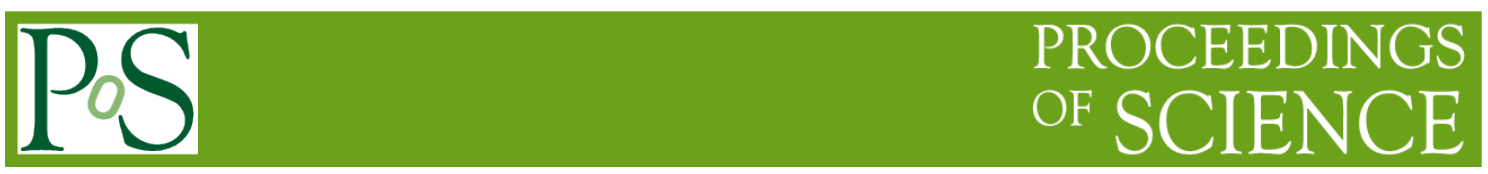

\title{
Design and realization of a vision system for Water Strider Robot
}

\author{
Licheng $\mathbf{W u}^{12}$ \\ School of Information Engineering, Minzu University of China , Beijing,100081, China \\ E-mail: wulichengetsinghua.edu.cn

\section{Yanming Fan, Xiaer Li} \\ School of Information Engineering, Minzu University of China , Beijing,100081, China
}

\begin{abstract}
In recent years, researches on water strider robot (WSR) which mimic water strider have become a new interested research direction in the field of bionic robotics. This paper presents a vision sensing system for water strider robot which uses circular footpads, This system uses a CMOS (Complementary Metal Oxide Semiconductor) image sensor to capture images, and sends images to the receiver specified by wireless communication module. The experimental tests show that the system can successfully fulfill the functions of taking photos and sending image via radio. Meanwhile, the experimental system is successfully realized with the feature of small size, light weight, and low power consumption, which ensure the system can be bear on robot.
\end{abstract}

\footnotetext{
${ }^{1}$ Speaker

${ }^{2}$ This work was supported in part by the transition funds of the first-class university and the first-class discipline construction, the 2013 Beijing university youth talent plan No. YETP1294, the NSFC projects No. 51375504, and the Program for New Century Excellent Talents in University.
} 


\section{Introduction}

Water Strider Robot is a kind of bionic robot of water strider that can stand and move on the water surface via the surface tension. At present, the study of WSR has made some research results, which can be achieved to walk, turn, or even jump on the water surface under laboratory conditions [1-2] but early stage water strider robot prototype load capability is limited, which can only take $5 \mathrm{~g}$ or less weight, so the vision sensing system is not designed to the robot. In 2010 year, water strider robot using circular footpads appeared, which greatly improved the robot load ability. The robot can take 53g weight [3], which maybe make WSR realize vision sensing come true. Imaging sensor is much significance for WSR to realize water quality monitoring, environmental monitoring and etc.

So far, there is no vision sensing system applied to WSR, but many studies about miniature vision sensing system have present on other tiny system. Due to the micro vision system is mainly applied on micro system, the system resources is limited, so the micro vision system's power consumption, volume and weight are greatly restricted. In 2003 year, a vision system was designed to MAVs named black widow [4-5], which uses CMOS image sensor and $2.4 \mathrm{GHz}$ wireless module to design vision system and realized compact size, light weight, but its larger power consumption is not suitable for coin battery. Mahlknecht etc present a real-time image recognition system, whose size is $35 * 35 \mathrm{~mm}$.It use DSP as processor and its power is $250 \mathrm{~mW}$, but wireless transmission module was not applied on it[6]. Jia etc present a kind of system that using FPGA as the core of the image acquisition and processing system [7], but its high power consumption does not meet the WSR low-power requirements. A method to use DSP as process center was designed by Xin and Sun [8], although it meets high image processing speed, its power does not meet the design requirements. These are also other researchers adopt MSP430 as microprocessor and transfers image date via GPRS [9]. Although it satisfy low power consumption demand, the transmission rate is slow, which cannot satisfy the requirement of real-time. Because the methods put forward by above scholars cannot meet WSR's requirements in some ways, such as consumption, size, weight function, image transmission real time, so we propose a new kind way using MSP430 microcontroller, CMOS image sensor and nRF24L01+ to realize WSR's vision sensing system. According to the results of performance evaluation and practical tests in the field, the proposed system has successfully accomplished the image acquisition and radio transmission tasks.

\section{System Structure Design}

Due to WSR's volume and support capabilities are limited, how to integrate drive, controllers, energy, sensors or actuators on the level of micro size and light weight become a key technology in designing robot. Therefore, the system structure design should take the robot overall weight into account, so that it will be light weight, energy-efficient and miniaturization.

In this paper, the visual sensor system mainly includes the following modules: micro control module, image sensor and storage module, wireless communication module and power module. Figure 1 is overall structure diagram for this system.

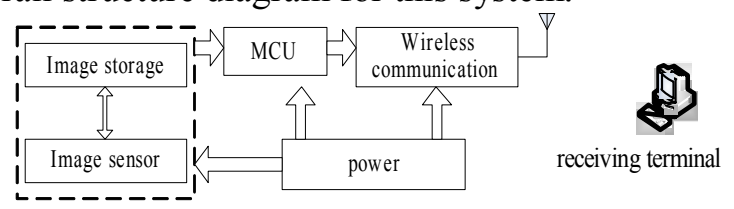

Figure 1: structure diagram of system

\subsection{Micro Controller Unit and Power Modules}

In view of facts that the robot is lightweight, power limited, a micro controller should meet the following basic requirements: 1. light weight, small package; 2 . rich on-chip resources; 3 . less peripheral components to drive it; 4 . low-power, low driving voltage; 5 . simply in design 
and mature software development environment and hardware simulation tools. Given the above conditions, The paper chooses TI's ultra-low-power chip MSP430F149 as the system master chip, which meets the conditions and simple in development and design。

In order to reduce the system weight, we can only use coin battery to provide energy. The system uses CR2032 type coin battery and its weight is only $3 \mathrm{~g}$. Due to image acquisition high power consumption, so we need voltage regulator design. On both ends of the battery, we connect some electrolytic capacitors.

\subsection{Image sensor and storage module}

So far, the image acquisition technique generally adopts CMOS and CCD sensors. Compared to CCD, the CMOS image sensor is low power consumption, low cost, and small size. Although the image quality CMOS image sensor obtained is relatively poor, considering the system power consumption, design costs and the image data transfer speed and other factors, this module chooses to use the CMOS image sensor.

We uses OmniVision's OV7670 image sensor. The image sensor chip's clock rate is $24 \mathrm{MHz}$, while MCU's I/O port rate can't match it, therefore, we consider using dynamic memory chip to solve the image sensor and MCU velocity mismatch, so that the image data can be sent to the radio transmission via memory module directly. We select AVERLOGIC company launched FIFO memory chip AL422B as dynamic storage, specific image acquisition module and $\mathrm{MCU}$ connection diagram is shown as Figure 2.

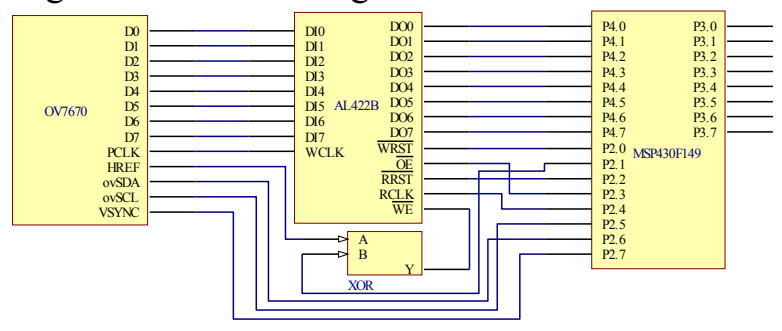

Figure 2: Connection diagram of image capture module and $\mathrm{MCU}$

OV7670's PLCK(pixel clock) pin connects to AL422B's WCK (write clock) pin. OV7670's field sync signal pin HREF and MSP430's P2.1 pin WEN generate FIFO write enable signal WE by XOR operation, so that the pixel information can be written to memory synchronously. OV7670 frame synchronization signal VSYNC trigger the MSP430 P2.7 pin's interrupt, then execute the interrupt programmer to read image data in memory.

\subsection{Wireless communication module}

The wireless communication module is important portion in the vision sensing system. Due to the information transmitted is image data, the data is so large and real-time high, therefore, requirements for this module are: 1 . small package, preferably PCB antenna; 2. lower power consumption; 3 . transmission faster; 4 .low cost. Considering the above requirements, this module selects NORIDC's high-speed, low-power, low-cost wireless communication chip nRF24L01+.The specific connection with the MCU module is shown as Fig 3. MSP430's P3 port control nRF24L01+ chip state. 


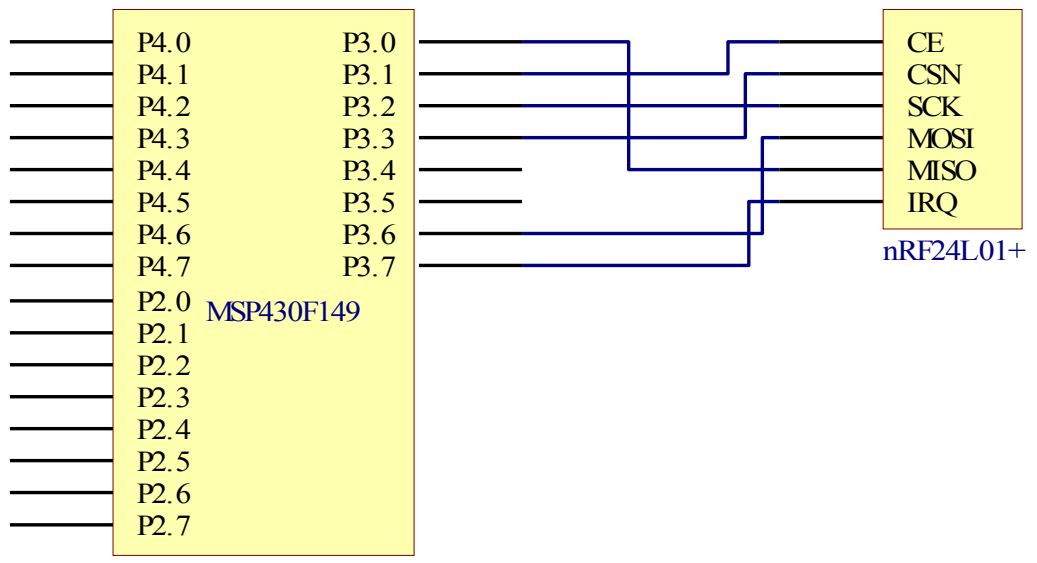

Figure 3: Connection diagram of nRF24L01+ and MCU

\section{System Software Design}

For the vision sensing system, we design an energy-efficient mechanism to control all operations. The mechanism consists of three processes. They are 1) system initialization, 2) image acquisition and transmission process, and 3) into ULP(Ultra Low Power) state, respectively. The first process completes OV7670 image sensor and nRF24L01+ registers setting, then start MSP430' interrupt. The second process implements interrupt service program to realize image acquisition and transmission. After the transmission of an image is finished, the OV7670 image and the radio modem will be turned off, then they are into the last process the ULP mode until the next interrupt is triggered. Figure 4 is system design process chart, the left side of the image is the overall process of visual sensing system, after completing system initialization and other modules work mode setting, the system will enter into the interrupt process when the system interrupt is triggered, as shown on the right side. When VSYNC is the first high level, open the memory write enabled signal WE, then write image data to storage. After the second high level change to low, the system completes to gain a image, then the image information is sent to the receiving side by the wireless communication module.
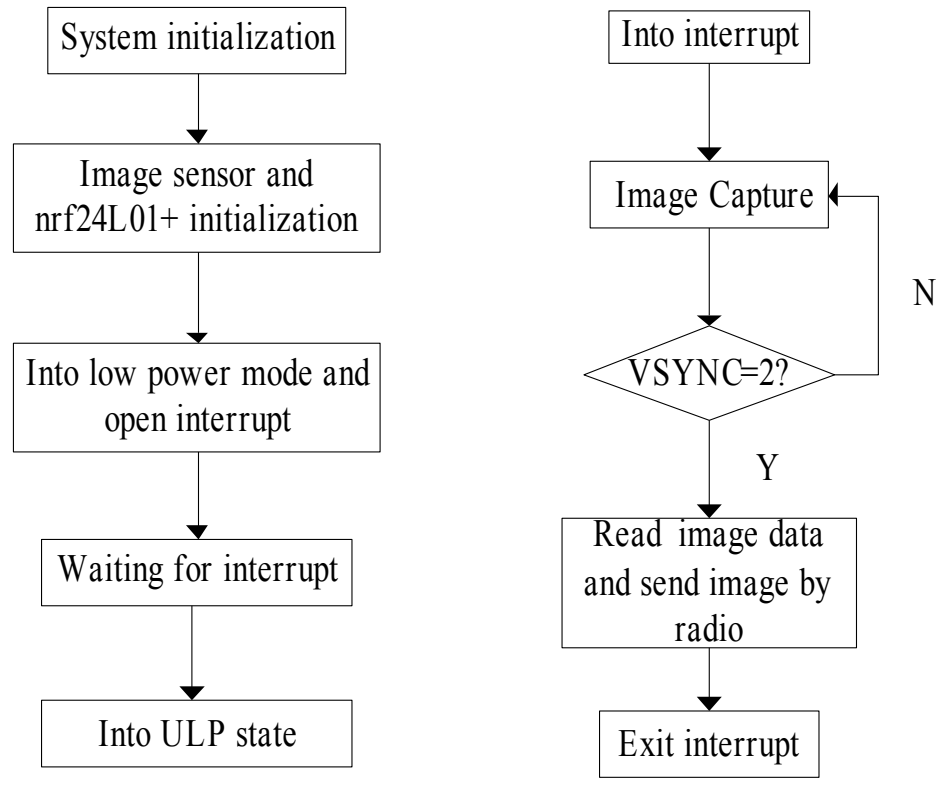

Figure 4: system software design chart 


\section{Experiments and Analyses}

In order to validate the system performance and effectiveness, the paper conducts experiments and analyses on the system from two aspects: power consumption and real-time of image transfer. The main energy-consuming devices are MSP430, OV7670, AL422B and nRF24L01+. Table 1 shows the energy consumption of each chip in the system during operation.

\begin{tabular}{llllll}
\hline \multirow{2}{*}{ Module } & \multicolumn{6}{l}{ Components work state and power } \\
& Ov7670 & AL422B & MCU & NRF24L01+ & Power \\
\hline Capture image & $\mathrm{w}^{1}$ & $\mathrm{~s}$ & $\mathrm{w}$ & $\mathrm{s}$ & $62.6 \mathrm{~mW}$ \\
Storage & $\mathrm{s}$ & $\mathrm{w}$ & $\mathrm{w}$ & $\mathrm{s}$ & $\mathrm{W}=30.6 \mathrm{mWR}=28.6 \mathrm{~mW}$ \\
Radio & $\mathrm{s}$ & $\mathrm{s}$ & $\mathrm{w}$ & $\mathrm{w}$ & $\mathrm{RX}=41.6 \mathrm{mWTX}=38.2 \mathrm{~mW}$ \\
MCU work & $\mathrm{s}$ & $\mathrm{s}$ & $\mathrm{w}$ & $\mathrm{s}$ & $6.3 \mathrm{~mW}$ \\
ULP & $\mathrm{s}$ & $\mathrm{s}$ & $\mathrm{s}$ & $\mathrm{s}$ & $84.5 \mathrm{uW}$ \\
\hline \multicolumn{1}{l}{ 1"w" denotes } & working "s" denotes shutdown
\end{tabular}

Table 1: the power consumption of components

System energy consumption is mainly determined by the working hours and working state of the chip. OV7670 working time is mainly determined by the image size and the sensor exposure time. NRF24L01+ wireless transmit image time-consuming and AL422B read and write operations time are also associated with image data size. It is shown as table 2 that the result of the vision sensing system proposed by this paper compared with other three systems.]

\begin{tabular}{llllll}
\hline System & $\begin{array}{l}\text { Power } \\
\text { consuming }\end{array}$ & Size & Weight & $\begin{array}{l}\text { Wireless } \\
\text { module }\end{array}$ & $\begin{array}{l}\text { Independent } \\
\text { power }\end{array}$ \\
\hline $\begin{array}{l}\text { This } \\
\text { paper }\end{array}$ & $140 \mathrm{~mW}$ & $40 * 60 \mathrm{~mm}$ & $11 \mathrm{~g}$ & Yes & Yes \\
Paper[5] & $700 \mathrm{~mW}$ & -- & $9 \mathrm{~g}$ & Yes & No \\
Paper[6] & $250 \mathrm{~mW}$ & $35 * 35 \mathrm{~mm}$ & -- & No & No \\
Paper[9] & $300 \mathrm{~mW}$ & $>>40 * 60 \mathrm{~mm}$ & $>>11 \mathrm{~g}$ & Yes & Yes \\
\hline
\end{tabular}

Table 2: the contrast result of system performent

It is found that WSR's vision system have advantage in power consuming, so that the system can work on coin battery condition.

In the image transmission time test, we varied $\mathrm{m}$ from 5 to 20 with an increment of 5 . Each case was tested 10 times, during each transmission of image, required transmission time were recorded. The image size is $320 * 240$ and the image format is RGB565. The length of the transmission packet is $32 \mathrm{~B}$, under $1 \mathrm{Mb} / \mathrm{s}$ transfer rate conditions. The result is shown as Fig 5 . The experiment test system is shown as Fig 6. 


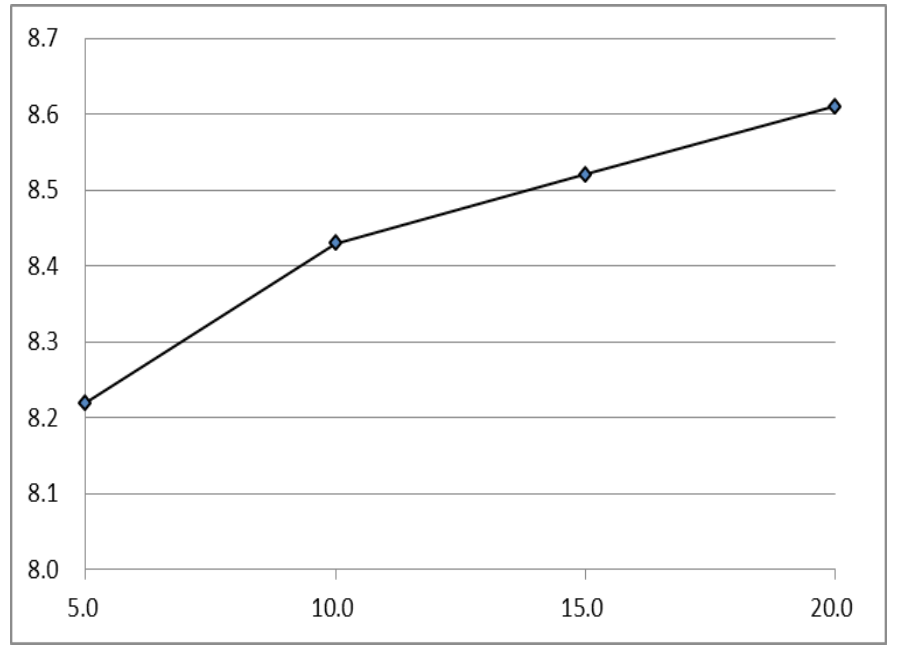

Figure 5: Image transmission time(second $/ \mathrm{m})$

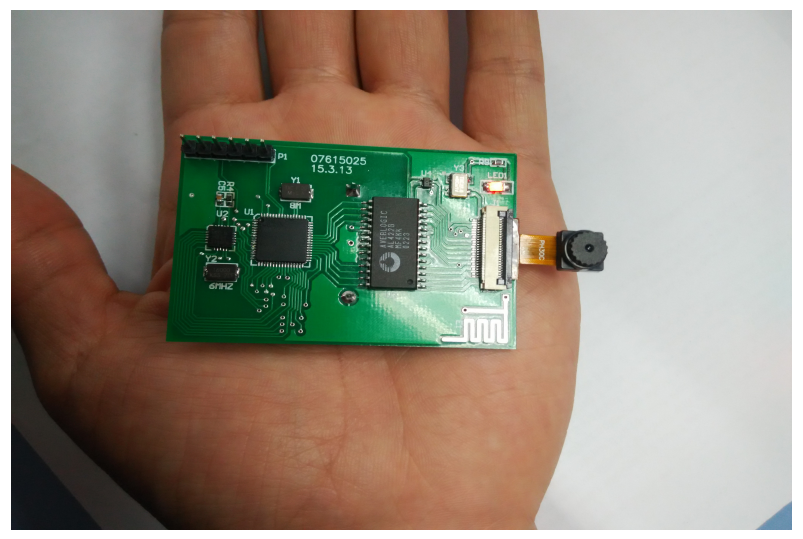

Figure 6: experiment test system.

From Fig.5, it can be observed that the longer the transmission distance, the longer transmission time, but the gap is very small. Why the image transmission time is about $10 \mathrm{~s}$, it is because that the MSP430 operating speed is slow, which cannot reach DSP or ARM chip, and the image information is not compressed.

\section{Conclusion}

A kind of vision sensing system is proposed for water strider robot which uses circular footpads. The vision system uses MSP430F149 chip cooperated with the OV7670 image sensor and DRAM AL422B to complete image acquisition. It also achieves peer to peer wireless image data transmission via wireless chip nRF24L01+. The experiment has showed that the vision sensing system can accomplish image acquisition and radio transmission. Also, it has many features, such as low power consumption, low design cost. But there are still some possible directions for us to improve the system. For example, integrating image compression technologies into the proposed system is possibility. With the technologies, the amount of transmitted data can be significantly reduced. Thus, the energy consumption and transmission time will also be drastically declined.

\section{References}

[1] S. H. Suhr, Y. S Song, S. J. Lee, M. Sitti. Biologically inspired miniature water strider robot [C]. Proceedings of the Robotics: Science and Systems I. Boston, USA: 2005: 319-325. 
[2] L. Wu, Z. Lian, G. Yang, M. Ceccarelli. Water Dancer II-a: a Non-tethered Telecontrollable Water Strider Robot[J]. International Journal of Advanced Robotic Systems, 2011, 8(4):10-17.

[3] X. Zhang. J. Zhao. Q. Zhu., N. Chen., M. Zhang, Q Pan. Bioinspired aquatic microrobot capable of walking on water surface like a water strider[J]. ACS Appl. Mater. Interfaces, 2011, 3(7):26302636.

[4] O. Ozcan, H. Wang, J. D. Taylor, M. Sitti. Surface tension driven water strider robot using circular footpads $[\mathrm{J}]$. Proceedings - IEEE International Conference on Robotics and Automation, 2010, 58(8):3799 - 3804.

[5] M. T. Keennon, J. M. Grasmeyer. Development of the Black Widow and Microbat MAVs and a Vision of the Future of MAV Design[C]. AIAA International Air and Space Symposium and Exposition: The Next 100 Years. Dayton, USA: 2003: 14-17.

[6] S. Mahlknecht, R. Oberhammer, G. Novak. A real-time image recognition system for tiny autonomous mobile robots[J]. Real-Time Systems, 2005, 29(2-3): 247-261.

[7] J. Jia, J. Y. Lai, G. H. Zhang, H. Ling. The Design of Image Acquisition and Preprocess SystemBased on FPGA[J] .Applied Mechanics and Materials,2011(71-78):4269-4273

[8] Y. Xin, X. Sun. System Design for Real-time Image Processing Based on Multi-core $D S P[\mathrm{~J}]$ Journal of Networks,2014(9):3143-3150

[9] C. P. Chen, C. H. Lin, T. W. Lai, C. L. Chuang, T. S. Lin, J. A. Jiang, C. H. Hong. EnergyEfficient Visual Eyes System for Wildlife[C]. //IEEE International Conference on High Performance Computing and Communications. IEEE, Banff,UK: 2011:869 - 874. 